\title{
High Density QCD Through Eletromagnetic Probes
}

\author{
M. A. Betemps ${ }^{1,2}$ and M. B. Gay Ducati ${ }^{1}$ \\ ${ }^{1}$ High Energy Physics Phenomenology Group, GFPAE \\ Instituto de Física, Universidade Federal do Rio Grande do Sul \\ Caixa Postal 15051, CEP 91501-970, Porto Alegre, RS, Brazil. \\ and \\ 2 Conjunto Agrotécnico "Visconde da Graça", CAVG \\ Universidade Federal de Pelotas \\ Caixa Postal 460, CEP 96060-290, Pelotas, RS, Brazil.
}

Received on 10 January, 2007

\begin{abstract}
In this work we investigate the high density QCD system through the dilepton production. First, the dilepton production in the color dipole approach is investigated, studing perturbative unitarity corrections to the dipole cross section and its consequence in the transverse momentum distribution of the dileptons at RHIC and LHC energies. Second, the dilepton production in the context of the Color Glass Condensate is investigated. The transverse momentum distribution and the rapidity distribution are investigated to dilepton production at RHIC and LHC energies in this framework.
\end{abstract}

Keywords: Color Glass Condensate; Dipole approach; Dilepton production

\section{INTRODUCTION}

The high energies hadronic reactions performed at RHIC (BNL Relativistic Heavy Ion Collider) and to be reached at LHC (CERN Large Hadron Collider) provide a suitable enviroment to investigate the dynamic of the high density system. In such a regime, the lepton pair production should be useful to investigate the high parton density at the initial state of the collision, once its carry indisturbed information about this stage of the reaction since does not interact strongly with the high density hadronic system, formed after the collision process.

This work intends to investigate the dilepton production in order to investigate the high density effects and is performed in two parts; in the first part an overview concerning the influence of perturbative unitarity corrections on the dilepton transverse momentum $p_{T}$ distribution is investigated, treating the dilepton production in the dipole approach, describing these unitarity corrections by the multiple scattering GlauberMueller approach [1]. A striking advantage of the color dipole picture is a finite cross section for the lepton pair $p_{T}$ distribution at small $p_{T} \rightarrow 0$, even in the leading order calculation, feature associated with the saturation encoded in the dipole cross section. The second part of this work, performs an investigation of the dilepton considering the Color Glass Condensate (CGC) approach and looking for the satuation signatures in dileptons produced at small transverse momentum in the forward rapidity region, or proton fragmentagion region.

The unitarity corrections, considering the dipole approach, are investigated in the proton-proton collisions, since the effect of perturbative corrections should be more evident in this reaction (the nuclear effects present in nuclear collisions should mask the unitiarty corrections), however, the dilepton production was shown to be a sensitive probe of the perturbative shadowing and saturation dynamics in all possibilities of reactions [2-12].

Considering the Color Glass Condensate kinematical regime, the saturation effects are investigated comparing the proton-nucleus and proton-proton collisions, since the saturated regime could be reached at RHIC only with nuclear collisions. Moreover, the proton-nucleus scattering was proposed as an ideal experiment to give evidences of the saturation effects described by the CGC in the proton fragmentation region [12]. In order to investigate the quantitative features of the dilepton production in the forward region of proton-nucleus collisions the transverse momentum $\left(p_{T}\right)$ distribution is studied focusing attention to the small $p_{T}$ region, where the saturation effects are expected to be more significant. Their presence at small $p_{T}$ should be considered as a possible signature of the saturation effects when contrasted with proton-proton results. This comparison is performed evaluating the ratio between proton-nucleus and proton-proton cross sections. This ratio, concerning hadron production, was measured at RHIC and at mid-rapitidy the ratio present a peak, called Cronin effect; at forward rapidities the ratio is suppressed, and is claimed to be due to the saturation effects. Concerning dileptons, this ratio shows two diferent behaviors at forward rapidities, it presents Cronin type peak or a suppression of the peak, depending of the dynamics considered for the Color Glass Condensate approach [13]. It implies that dilepton should be a most suitable probe to identify the saturation effects at high energy nuclear collisions.

This work is organized as follows. In the next section one presents a brief discussion on the dilepton production in the dipole picture. In Sec. III the results concerning the unitarity corrections are discussed. In Sec. IV the dilepton production within the CGC formalism is presented. The Sec. V presents the numerical results concerning the CGC.

\section{DILEPTON IN THE DIPOLE APPROACH}

The dipole approach is applicable only at high energies, and it is formulated in the target rest frame, where the dilepton production looks like a bremsstrahlung of a virtual photon de- 
caying into a lepton pair with a mass $M$ (see Fig. 1).

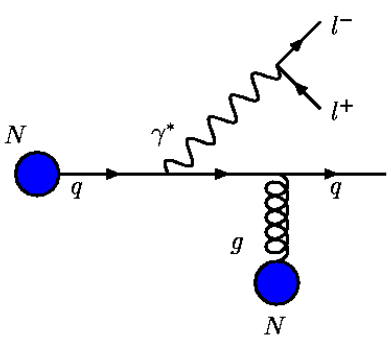

FIG. 1: Dilepton production in the dipole approach.

The advantage of this formalism is that the dilepton cross section can be written in terms of the same color dipole cross section as small- $x$ Deep Inelastic Scattering (DIS). Although diagrammatically no dipole is present in bremsstrahlung, the dipole cross section arises when one takes the complex conjugate of the amplitude (photon emited before or after the interaction with the target), see Ref. [14] for a detailed derivation.

The cross section of the dilepton production considering a proton-proton collision can be written as $[3,4]$

$\frac{d \sigma^{D Y}}{d M^{2} d x_{F} d^{2} p_{T}}=\frac{\alpha_{\mathrm{em}}^{2}}{6 \pi^{3} M^{2}} \frac{1}{\left(x_{1}+x_{2}\right)} \int_{0}^{\infty} d \rho W\left(\rho, p_{T}\right) \sigma_{d i p}\left(\rho, x_{2}\right)$,

where $x_{F}$ is the Feynman $x$ defined by $x_{F}=x_{1}-x_{2}, x_{1}$ and $x_{2}$ are the Bjorken $x$ defined in the standard way. $p_{T}$ is the transverse momentum of the dilepton and $\rho$ is the displacement of the projectile quark in impact parameter space due to the radiation of the virtual photon. The function $W\left(\rho, p_{T}\right)$ is given by

$$
\begin{aligned}
& W\left(\rho, p_{T}\right)=\int_{x_{1}}^{1} \frac{d \alpha}{\alpha^{2}} \frac{x_{1}}{\alpha} F_{2}^{p}\left(\frac{x_{1}}{\alpha}, M^{2}\right) \\
& \times \quad\left\{\left[m_{q}^{2} \alpha^{4}+2 M^{2}(1-\alpha)^{2}\right]\left[\frac{1}{p_{T}^{2}+\eta^{2}} T_{1}(\rho)-\frac{1}{4 \eta} T_{2}(\rho)\right]\right. \\
& \left.+\quad\left[1+(1-\alpha)^{2}\right]\left[\frac{\eta p_{T}}{p_{T}^{2}+\eta^{2}} T_{3}(\rho)-\frac{T_{1}(\rho)}{2}+\frac{\eta}{4} T_{2}(\rho)\right]\right\}
\end{aligned}
$$

where $\alpha$ is the light-cone momentum fraction of the intial quark taken away by the photon, $\eta^{2}=(1-\alpha) M^{2}+\alpha^{2} m_{q}^{2}$, with $m_{q}$ being the quark mass. The functions $T_{i}$ read,

$$
\begin{aligned}
& T_{1}(\rho)=\rho J_{0}\left(p_{T} \rho / \alpha\right) K_{0}(\eta \rho / \alpha) / \alpha, \\
& T_{2}(\rho)=\rho^{2} J_{0}\left(p_{T} \rho / \alpha\right) K_{1}(\eta \rho / \alpha) / \alpha^{2}, \\
& T_{3}(\rho)=\rho J_{1}\left(p_{T} \rho / \alpha\right) K_{1}(\eta \rho / \alpha) / \alpha .
\end{aligned}
$$

The functions $J_{0}$ and $J_{1}$ are the first class Bessel functions of order 0 and 1, whereas $K_{0}$ and $K_{1}$ are the second class modified Bessel functions of order 0 and 1 (MacDonald functions). The function $W\left(\rho, p_{T}\right)$ is originated from the wave function for radiation of a transversely or longitudinally polarized photon from the quark of the projectile. The structure function $F_{2}^{p}$ presented in the equation 2 take into account the quark content from the projectile. The function $\sigma_{\text {dip }}\left(\rho, x_{2}\right)$ is the dipole cross section and take into accout the interaction of the dipole with the target. This dipole cross section is the same obtained in the Deep Inelastic Scattering

As can be seen from Eq. (2), the oscillating Bessel functions $J_{i}$ drive the behavior of the $W\left(\rho, p_{T}\right)$ as a function of $\rho$. The following general picture can be drawn: for large $p_{T}$ the large dipole size configurations get suppressed, because $W\left(\rho, p_{T}\right)$ is rapidly oscillating. On the other hand, as $p_{T}$ decreases, large $\rho$ configurations become more important. The case $p_{T}=0$ is of particular interest, since the weight function $W\left(\rho, p_{T}\right)$ selects very large dipole configurations and such a region is enhanced by increasing the energy. Therefore, the non-perturbative sector of the process should drive the small $p_{T}$ regime. On the other hand, the large $p_{T}$ behavior is almost completely dominated by small dipole configurations [4] (perturbative contributions). The unitarity perturbative corrections are included in the dipole cross section and investigated in the transverse momentum distribution of the dileptons. It implies that only at large $p_{T}$ the perturbative correction should be more evident. These features are exploited in the next section, where are also discussed the different models that were employed for the dipole cross section.

\section{A. The dipole cross section}

In this section we consider the cross section of a color dipole interacting with a nucleon. The cross section for a small color dipole scattering on a nucleon can be obtained from perturbative QCD [15]. However, there are large uncertainties stemming from non-perturbative effects (infrared region) as well as from higher order and higher twist corrections. In the leading $\ln (1 / x)$ approximation, the dipole interacts with the target through the exchange of a perturbative Balitsky-FadinKuraev-Lipatov (BFKL) Pomeron, described in terms of the ladder diagrams [16]. In the double logarithmic approximation, the BFKL equation [16] agrees with the evolution equation of Dokshitzer et al. [17] (hereafter DGLAP equation). In this limit, the dipole cross section reads,

$$
\sigma_{d i p}\left(x, r_{\perp}\right)=\frac{\pi^{2} \alpha_{s}}{3} r_{\perp}^{2} x G^{\mathrm{DGLAP}}\left(x, \tilde{Q}^{2}\right)
$$

where $x G^{\text {DGLAP }}\left(x, \tilde{Q}^{2}\right)$ is the usual DGLAP gluon distribution at momentum fraction $x$ and virtuality scale $\tilde{Q}^{2}=\lambda / r_{\perp}^{2}$. In this work one considers the factor $\lambda=4$, although same magnitude values are equivalent at leading logarithmic level [18]. The main feature of the dipole cross section above is the color transparency property, i.e., $\sigma_{d i p} \sim r_{\perp}^{2}$ as $r_{\perp} \rightarrow 0$. At large dipole size, the dipole cross section should match the confinement property $\sigma_{d i p} \sim \sigma_{0}$. Concerning the large transverse separation (non-perturbative sector), our procedure is to freeze the $r_{\perp}^{2}$ in Eq. (6) at a suitable scale larger than $r_{\text {cut }}^{2}$, which corresponds to the initial scale on the gluon density perturbative evolution, $Q_{0}^{2}=4 / r_{\text {cut }}^{2}$.

At high energies, an additional requirement should be met: the growth of the parton density (mostly gluons) has to be tamed, since an uncontrolled increasing would violate the Froissart-Martin bound, requiring the black disc limit of the 
target has to be reached at quite small Bjorken $x$. This feature can be implemented by using the multiple scattering GlauberMueller approach (GM), which reduces the growth of the gluon distribution by eikonalization in impact parameter space [1]. Therefore, one substitutes $x G^{\text {DGLAP }}$ in Eq. (6) by the corrected distribution including unitarity effects, $x G^{G M}$. A more extensive derivation of the GM dipole cross section and the expression of $x G^{G M}$ can be found in the Sec. III of the Ref. [2]. Our main goal in this part of the work is to investigate the dilepton $p_{T}$ distribution, using the GM dipole cross section. However, for sake of comparison, this analysis is contrasted with the phenomenological saturation model of Bartels et al. (BGBK dipole cross section hereafter), Ref. [19], which also includes the features of the dipole cross section discussed above. The model of Ref. [19] is a QCD improved version of the saturation model of Ref. [20]. The new model explicitly includes QCD evolution, and the dipole cross section is given by,

$$
\sigma_{d i p}\left(x, r_{\perp}\right)=\sigma_{0}\left\{1-\exp \left(-\frac{\pi^{2} r_{\perp}^{2} \alpha_{s}\left(\mu^{2}\right) x g\left(x, \mu^{2}\right)}{3 \sigma_{0}}\right)\right\},
$$

where the scale $\mu^{2}$ is assumed to have the form $\mu^{2}=\frac{C}{r^{2}}+\mu_{0}^{2}$. The authors of Ref. [19] propose the following gluon distribution at initial scale $Q_{0}^{2}=1 \mathrm{GeV}^{2}$,

$$
x g\left(x, Q_{0}^{2}\right)=A_{g} x^{-\lambda_{g}}(1-x)^{5.6}
$$

Altogether, there are five free parameters $\left(\sigma_{0}, C, \mu_{0}^{2}, A_{g}\right.$ and $\lambda_{g}$ ), which have been determined in Ref. [19] by fitting ZEUS, $\mathrm{H} 1$ and E665 data with $x<0.01$. In this fit the parameter $\sigma_{0}$ is fixed at $23 \mathrm{mb}$ during the fits as in the original model, Ref. [20]. Here, we employ fit 1 of Ref. [19].

In Ref. [14], where the old saturation model of Ref. [20] was used, the dipole approach was extrapolated to larger $x_{2}$ by introducing a threshold factor into the saturation scale, i.e. $Q_{s}^{2} \rightarrow Q_{s}^{2}\left(1-x_{2}\right)^{5}$. The factor $\left(1-x_{2}\right)^{5}$ is motivated from QCD counting rules and suppresses the large $x_{2}$ contribution in the DY cross section. In our case, employing the GM or the BGBK dipole cross section, the large $x_{2}$ threshold factor is already included in the collinear gluon distribution function.

\section{B. The dilepton transverse momentum distribution}

In this section, the dilepton transverse momentum distribution is calculated, using the Glauber-Mueller dipole cross section, Eq. (6), and compared with the results obtained with the improved saturation model, Eq. (7). We will consider typical values for mass and $x_{F}$. The projectile structure function employed was the LO GRV98 parametrization [21] to the GM predictions and CTEQ5L [22] for the saturation model ones.

In Fig. 2 results for the energies from RHIC $(\sqrt{s}=500$ $\mathrm{GeV})$ and $\mathrm{LHC}(\sqrt{s}=14 \mathrm{TeV})$ are shown with $M=6.5 \mathrm{GeV}$ and $x_{F}=0.625$. We emphasize that the $x_{F}$ value considered above, is an extreme case, where the rapidity variable acquires large values for RHIC $(y \sim 3)$ and LHC $(y \sim 7)$ energies. In order to investigate the unitarity effects for this
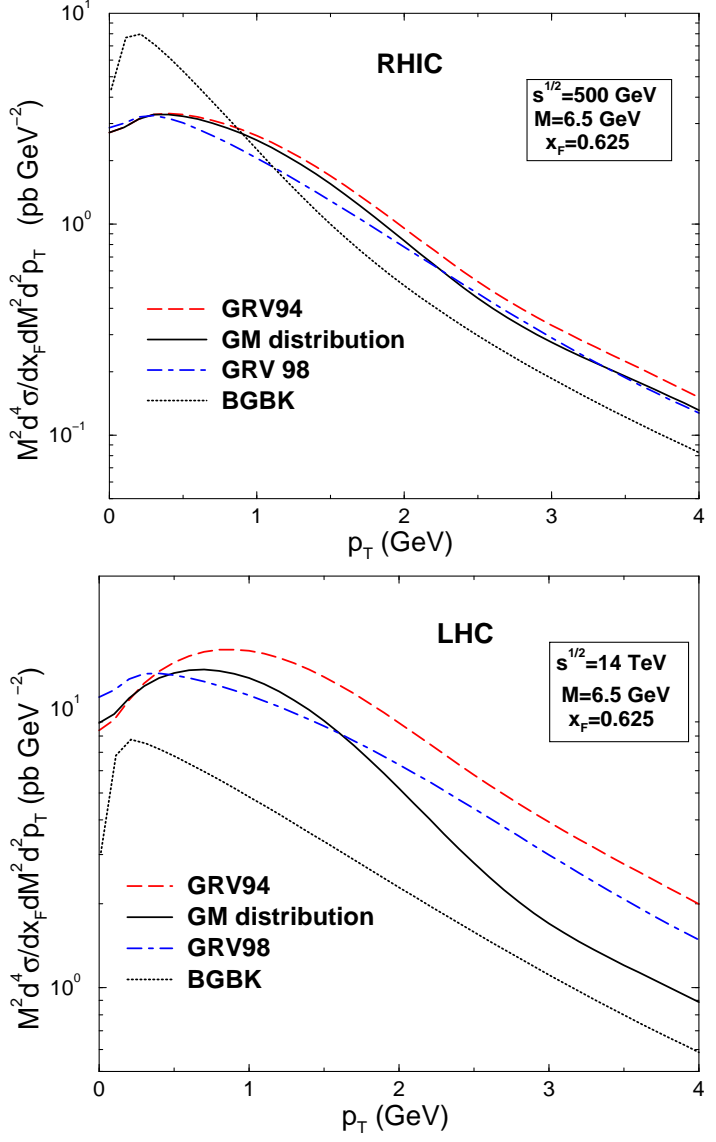

FIG. 2: The dilepton transverse momentum $p_{T}$ distribution at largest RHIC $(\sqrt{s}=500 \mathrm{GeV})$ and LHC $(\sqrt{s}=14 \mathrm{TeV})$ energies.

observable, the following comparisons are performed: The long-dashed curves are calculated with the dipole cross section, Eq. (6), without unitarity effects (denoted GRV94) using the GRV94 LO parameterization [23] in calculating the dipole cross section. The solid curves are the result including unitarity effects with the same GRV94 parameterization as initial input. The use of this parameterization is justified properly in Refs. [2, 24]. The dot-dashed curves are calculated with the dipole cross section, Eq. (6), using as input the GRV98 parameterization for the gluon structure function. The aim of this comparison is to verify to what extent an updated parameterization can absorb unitarity effects. It is verified that at RHIC energy, the unitarity effects could be absorbed in the parameterization. However, at LHC energy the situation is quite different, and the results are completely distinct. The deviation is important mostly at large $p_{T}$, once perturbative correction are under investigation. The unitariy corrections are enhanced as the energy increases.

\section{DILEPTON PRODUCTION IN THE COLOR GLASS CONDENSATE}

The Color Glass Condensate (CGC) picture holds in a frame in which the hadron propagates at the speed of light and, 
by Lorentz contraction, appears as a thin two-dimensional sheet located at the light cone. The formalism supporting this picture is in terms of a classical effective theory valid at small $x$ region (large gluon density), and was originally proposed to describe the gluons in large nuclei [25].

In this theory, the small $x$ gluons are radiated from fast moving color sources, which are partons with larger values of $x$, being described by a color source density $\rho_{a}$ with internal dynamics frozen by Lorentz time dilatation, thus forming a color glass. The observables are obtained by means of an average over all configurations of the color sources, performed through a weight functional $W_{\Lambda^{+}}[\rho]$, which depends upon the dynamics of the fast modes, and upon the intermediate scale $\Lambda^{+}$, which defines fast $\left(p^{+}>\Lambda^{+}\right)$and soft $\left(p^{+}<\Lambda^{+}\right)$modes. The effective theory is valid for soft momenta of order $\Lambda^{+}$. Indeed, reaching much softer scale, there are large radiative corrections which invalidate the classical approximation. The modifications to the effective classical theory are governed by a functional, nonlinear, evolution equation JIMWLK [26, 27] for the statistical weight functional $W_{\Lambda^{+}}[\rho]$ associated with the random variable $\rho_{a}(x)$.

In this approach, the diagrams that contributes to the dilepton production are the same considered in the dipole approach. In this way, the hadronic cross section for the dilepton production is obtained using the collinear factorization and considering the forward rapidity region $[9,13]$,

$$
\begin{aligned}
& \frac{d \sigma^{p A \rightarrow q l^{+} l^{-} X}}{d p_{T}^{2} d M d y}=\frac{4 \pi^{2}}{M} R_{A}^{2} \frac{\alpha_{e m}^{2}}{3 \pi} \\
& \quad \times \int \frac{d l_{T}}{(2 \pi)^{3}} \quad l_{T} \mathcal{W}\left(p_{T}, l_{T}, x_{1}\right) C\left(l_{T}, x_{2}, A\right),
\end{aligned}
$$

with $y$ being the rapidity, $s$ the squared center of mass energy, $l_{T}$ the total transverse momentum transfer between the nucleus and the quark and $R_{A}$ the nuclear radius. The expression $(9)$ is restricted to the forward region only, which means positive rapidities $y$ (or positive $x_{F}$ ). The function $\mathcal{W}\left(p_{T}, l_{T}, x_{1}\right)$ can be written as [9],

$$
\begin{aligned}
& \mathcal{W}\left(p_{T}, l_{T}, x_{1}\right)=\int_{x_{1}}^{1} d z z F_{2}\left(x_{1} / z, M^{2}\right) \\
& \times\left\{\frac{\left(1+(1-z)^{2}\right) z^{2} l_{T}^{2}}{\left[p_{T}^{2}+M^{2}(1-z)\right]\left[\left(p_{T}-z l_{T}\right)^{2}+M^{2}(1-z)\right]}\right. \\
- & z(1-z) M^{2}\left[\frac{1}{\left[p_{T}^{2}+M^{2}(1-z)\right]}\right. \\
- & \left.\left.\frac{1}{\left[\left(p_{T}-z l_{T}\right)^{2}+M^{2}(1-z)\right]}\right]^{2}\right\},
\end{aligned}
$$

which selects the values of $l_{T}$ larger than $p_{T}$ [13] Here, $F_{2}\left(x_{1} / z, Q^{2}\right)$ is the partonic structure function, which takes into account the quark distribution of the proton projectile and $z \equiv p^{-} / k^{-}$(light-cone variables) is the energy fraction of the proton carried by the virtual photon. In the Eq. 9 the function $C\left(l_{T}, x_{2}, A\right)$ is the field correlator function which, disregarding the energy and nuclear dependence, can be defined by [28],

$$
C\left(l_{T}\right) \equiv \int d^{2} x_{\perp} e^{i l_{T} \cdot x_{\perp}}\left\langle U(0) U^{\dagger}\left(x_{\perp}\right)\right\rangle_{\rho},
$$

with the averaged factor representing the average over all configurations of the color fields sources in the nucleus, $U\left(x_{\perp}\right)$ is a matrix in the $S U(N)$ fundamental representation which represents the interactions of the quark with the classical color field of the nucleus. All the information about the nature of the medium crossed by the quark is included in the function $C\left(l_{T}, x_{2}, A\right)$. In particular, it determines the dependence on the saturation scale $Q_{s}$ (and on energy), implying that all saturation effects are encoded in this function. In the Ref. [13] we have shown that the saturation effects appear in the function $C\left(l_{T}, x_{2}, A\right)$ only at small $l_{T}$, and as discussed here, the function $\mathcal{W}\left(p_{T}, l_{T}, x_{1}\right)$ selects values of $l_{T}$ larger than $p_{T}$, implying that only dileptons with $p_{T}$ smaller than $Q_{S}$ should carry information about the CGC. Regarding the structure function $F_{2}\left(x_{1} / z, M^{2}\right)$, the CTEQ6L parametrization [29] was used and the lepton pair mass gives the scale for the projectile quark distribution. Here some comments are in order; the cross section obtained in this approach is very similar to that obtained in the dipole picture. Indeed, the dilepton production in the CGC approach is a dipole approach, in the momentum space, with a particular configuration to the nuclear (cilindrical one).

The energy dependence introduced in the Eq. (9) in the correlator function $C\left(l_{T}, x_{2}, A\right)$ is performed by means of the saturation scale $Q_{s}\left(x_{2}, A\right)$. We employed the GBW parametrization [20] to obtain the $x$ dependence of the saturation scale $\left(Q_{s}^{2}=(x)\left(x_{0} / x\right)^{\lambda}\right)$, and the parameters have been taken from the dipole cross section extracted from the fit procedure by CGCfit [30] parametrization. The nuclear radius, which appears in the Eq. (9), is taken from the parametrization which has the form, $R_{A}=1.2 A^{1 / 3} \mathrm{fm}$, while the proton radius (for $p p$ calculations) is taken from the fit [30] $\left(R_{p}=0.6055 \mathrm{fm}\right)$.

In order to obtain numerically the dilepton differential cross section, the function $C\left(l_{T}, x, A\right)$ need to be specified. The simplest model to this function should be a local Gaussian, however, it was verified that this consideration implies in a nonrealistic description of the RHIC experimental results concerning the hadron transverse momentum distribution [31]. In this work we have evaluated the cross section using the function $C\left(l_{T}, x, A\right)$ from the mean-field asymptotic solution for the JIMWLK evolution equation [32], introducing an $x$ dependence through the nuclear saturation scale [13], which is parametrized in the form $Q_{s}^{2}(x, A)=A^{1 / 3} Q_{s}^{2}(x)$. These considerations imply for the correlator the following form $[31,33]$

$$
C\left(l_{T}, x, A\right) \equiv \int d^{2} x_{\perp} e^{i l_{T} \cdot x_{\perp}} e^{\chi\left(x, x_{\perp}, A\right)}
$$

with

$$
\begin{aligned}
\chi\left(x, x_{\perp}, A\right) & \equiv-\frac{2}{\gamma c} \int \frac{d p}{p}\left(1-J_{0}\left(x_{\perp} p\right)\right) \\
\times & \ln \left(1+\left(\frac{Q_{2}^{2}(x, A)}{p^{2}}\right)^{\gamma}\right),
\end{aligned}
$$

where, $\gamma$ is the anomalous dimension $(\gamma \approx 0.64$ for BFKL) and $c \approx 4.84[31,33]$. 


\section{A. Saturation effects in dilepton production}

As discussed in the introduction of this work, the Cronin effect is present in the measurement of the hadron transverse momentum spectra. Here, the onset of the same effects in the dilepton $p_{T}$ and rapidity spectra is investigated for a lepton pair mass $M=6 \mathrm{GeV}$. In a previous work [13], the investigation was performed for the $p_{T}$ distribution of dileptons with mass $M=3 \mathrm{GeV}$. Now, one evaluates the $p_{T}$ and rapidity spectra for the dilepton at RHIC and LHC energies, $\sqrt{s}=200$ $\mathrm{GeV}$ and $\sqrt{s}=8800 \mathrm{GeV}$, respectively.

We have defined the nuclear modification ratio for the dilepton production in the following form,

$$
R_{p A}=\frac{\frac{d \sigma(p A)}{\pi R_{A}^{2} d M d y d p_{T}^{2}}}{A^{1 / 3} \frac{d \sigma(p p)}{\pi R_{p}^{2} d M d y d p_{T}^{2}}} .
$$

Some attention should be given to the uncertainty in the determination of the nuclear radius, then each cross section is divided by the nuclear or proton radius. The factor $A^{1 / 3}$ was used in the denominator to guarantee a ratio $R_{p A}$ about 1 at large $p_{T}$.

The comparison between the results for the ratio $R_{p A}$ considering two distinct lepton pair masses can be performed and the effect of the suppression of the ratio is reduced if the dilepton mass is increased at a fixed rapidity [34]. Such result can be verified in the Ref. [6], where an analysis was performed for the dilepton production at RHIC and LHC energies in the color-dipole formalism, however, there the ratio was defined with a different normalization.

This analysis regards forward rapidities, which maximum value depends on the value of the mass and the transverse momentum. The region of large mass and large $p_{T}$ implies smallest values for the rapidity limit. At RHIC energies the maximum rapidity value reaches 4 and at LHC energies it goes up to 7 .

In the Fig. 3 the nuclear modification ratio for RHIC energies is shown for dilepton mass $M=6 \mathrm{GeV}$. A weak dependence of the ratio $R_{p A}$ with the rapidity range is verified, since for a fixed $p_{T}$ value, the ratio does not vary significantly with rapidity. This occurs due to the fact that one evaluates the ratio $R_{p A}$ only at forward rapidities, in a short limited range. For the hadron spectra, the suppression of the ratio with the increase of the rapidity is verified for a large range of rapidities, from $y=0$ to forward ones $(y=3.2)$ [35]. In the case of the dileptons, the same suppression should be verified, however the calculation is restricted to the forward rapidities, providing a small suppression in the rapidity range investigated here. The suppression of the ratio (absence of a Cronin type peak) in the $p_{T}$ distribution is verified, although independent of the rapidity. Our results are similar to the obtained in the Ref. [8] for RHIC energies with dilepton mass $M=2$ and $4 \mathrm{GeV}$. There, the ratio $R_{p A}$ presents smaller values in the $p_{T}$ spectra at large rapidities.

In the Fig. 4 the nuclear modification ratio for LHC energies is shown for dilepton mass $M=6 \mathrm{GeV}$. Due to the large range of forward rapidities at LHC energies, one verifies the

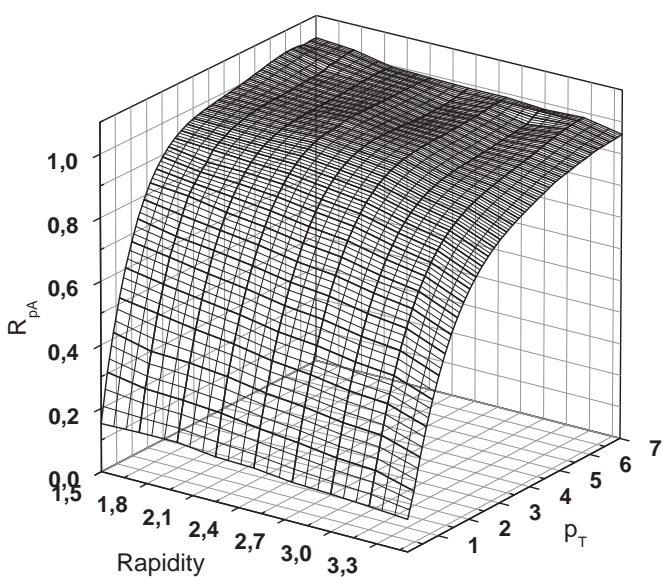

FIG. 3: Ratio $R_{p A}$ as a function of rapidity and $p_{T}$ for dileptons at RHIC energies.

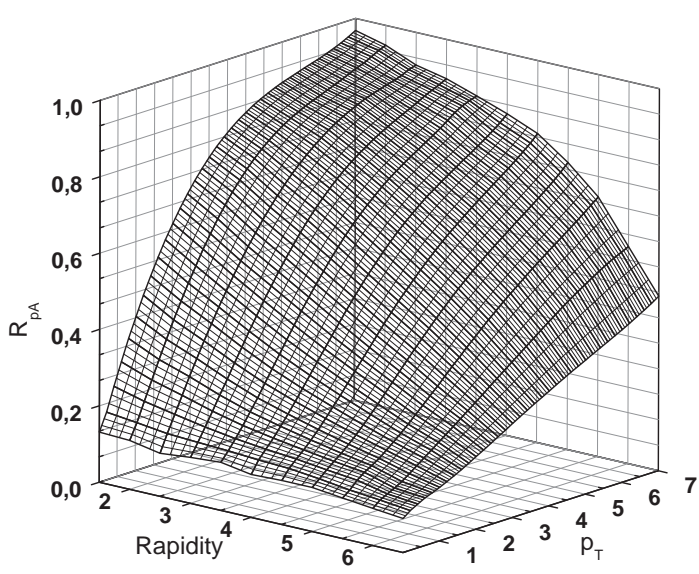

FIG. 4: Ratio $R_{p A}$ as a function of rapidity and $p_{T}$ for dileptons at LHC energies.

large suppression of the nuclear modification ratio with the increase of the rapidity. This suppression is intensified at large $p_{T}$. The suppression of the same ratio with the transverse momentum is also verified and is intensified at large rapidities.

At LHC kinematical region, there are significant effects of saturation predicted by the Color Glass Condensate: the large suppression of the nuclear modification ratio comparing with the expected Cronin peak shows the existence of these effects, in both, rapidity and transverse momentum distributions.

The predicted ratio for RHIC and LHC energies is evaluated considering the same description for both, nucleus and nucleons. This implies that the nucleon is not well described, since at intermediated $p_{T}$ the nucleon is in the linear regime, and we 
are using a non-linear approach to the proton. This simplification provides some uncertainty in the ratio at large $p_{T}$, mainly at RHIC energies, where the proton saturation scale reaches small values. At LHC energies, the uncertainty in the ratio at large $p_{T}$ is reduced, since the saturation scale of the proton reaches larger values. The effect of considering a realistic proton appears as a small reduction of the ratio $R_{p A}$ at large $p_{T}$, since there is no large suppression in proton gluon density in the linear regime. However, a realistic description of the proton requires the determination of a new parametrization, which is out of the scope of this work.

\section{CONCLUSIONS}

In this work the dilepton production was shown to probe perturbative unitarity corrections at large $p_{T}$ and at the same time, provides information about the dense saturated regime (Color Glass Condensate) at the small $p_{T}$. The unitarity perturbative corrections were investigated in the dipole approach, while the saturation effects were investigated considering the CGC approach. The $p_{T}$ distribution was shown to be a powerful distribution to seeking unitarity and saturation effects. The rapidity spectra provides another distribution useful to investigate saturation effects, mainly at forward ones. These features qualify dileptons as a cleanest probe to the Color Glass Condensate dynamics at forward rapidities and of the unitarity corrections.

Acknowledgments: We are greatful to all participants of the meeting due to the excelent discussions during the ILAWHEP. This work was supported by CNPq.
[1] A.L. Ayala, M.B. Gay Ducati, and E.M. Levin, Nucl. Phys. B 493, 305 (1997); Nucl. Phys. B 511, 355 (1998).

M.B. Gay Ducati, V.P. Gonçalves, Nucl. Phys. B 557, 296 (1999); Nucl. Phys. (Proc. Suppl.) 79, 302 (1999).

[2] M.A. Betemps, M.B. Gay Ducati, and M.V.T. Machado, Phys. Rev. D 66, 014018 (2002).

M.A. Betemps, M.B. Gay Ducati, and M.V.T. Machado, In proceedings 8th International Workshop on Hadron Physics 2002. Topics on the Structure and Interaction of Hadronic Systems, Bento Gonçalves, Brazil, p. 323, (2003).

[3] M.A. Betemps, M.B. Gay Ducati, M.V.T. Machado, and J. Raufeisen, Phys. Rev. D 67, 114008 (2003).

[4] B.Z. Kopeliovich, J. Raufeisen, and A.V. Tarasov, Phys. Lett. B 503, 91 (2001).

[5] B.Z. Kopeliovich, In proceedings Workshop Hirschegg'95: Dynamical Properties of Hadrons in Nuclear Matter. Ed. by $\mathrm{H}$. Feldmeier and W. Nörenberg, GSI, Darmstadt, p. 102 (1995) [hep-ph/9609385];

S.J. Brodsky, A. Hebecker, and E. Quack, Phys. Rev. D 55 2584 (1997).

[6] B. Z. Kopeliovich, J. Raufeisen, A. V. Tarasov, and M. B. Johnson, Phys. Rev. C 67, 014903 (2003).

[7] G. Fai, J. w. Qiu, and X. f. Zhang, J. Phys. G 30, S1037 (2004).

[8] R. Baier, A. H. Mueller, and D. Schiff, Nucl. Phys. A 741, 358 (2004).

[9] F. Gelis, J. Jalilian-Marian, Phys. Rev. D 66, 094014 (2002).

[10] F. Gelis, J. Jalilian-Marian, Phys. Rev. D 67, 074019 (2003).

[11] J. Jalilian-Marian, Nucl. Phys. A 739, 319 (2004).

[12] A. Dumitru and J. Jalilian-Marian, Phys. Rev. Lett. 89, 022301 (2002)

[13] M.A. Betemps, M.B. Gay Ducati, Phys. Rev. D 70, 116005 (2004); Eur. Phys. J. C 43, 365 (2005).

[14] J. Raufeisen, J.C. Peng, and G.C. Nayak, Phys. Rev. D 66, 034024 (2002).

[15] B. Blättel, G. Baym, L. L. Frankfurt, and M. Strikman, Phys. Rev. Lett. 70, 896 (1993);

L. Frankfurt, A. Radyushkin, and M. Strikman, Phys. Rev. D 55, 98 (1997).

[16] E.A. Kuraev, L.N. Lipatov, and V.S. Fadin, Phys. Lett. B 60, 50 (1975); Sov. Phys. JETP 44, 443 (1976); Sov. Phys. JETP 45, 199 (1977);
Ya. Balitsky and L.N. Lipatov, Sov. J. Nucl. Phys. 28, 822 (1978).

[17] Yu. L. Dokshitzer. Sov. Phys. JETP 46, 641 (1977); G. Altarelli and G. Parisi. Nucl. Phys. B 126, 298 (1977); V.N. Gribov and L.N. Lipatov. Sov. J. Nucl. Phys 28, 822 (1978).

[18] M. McDermott et al., Eur. Phys. J. C 16, 641 (2000). L. Frankfurt, M. McDermott, and M. Strikman, JHEP 0103, 45 (2001).

[19] J. Bartels, K. Golec-Biernat, and H. Kowalski, Phys. Rev. D 66, 014001 (2002)

[20] K. Golec-Biernat, M. Wüsthoff, Phys. Rev. D 59, 014017 (1999); Phys. Rev. D 60, 114023 (1999).

[21] M. Glück, E. Reya, and A. Vogt, Eur. Phys. J. C 5, 461 (1998).

[22] H. L. Lai et al. [CTEQ Collaboration] Eur. Phys. J. C 12, 375 (2000).

[23] M. Glück, E. Reya, and A. Vogt, Z. Phys. C 67, 433 (1995).

[24] M.B. Gay Ducati, M.V.T. Machado, Phys. Rev. D 65, 114019 (2002).

[25] L. McLerran, R. Venugopalan, Phys. Rev. D 49, 2233 (1994); ibid. 49, 3352 (1994).

[26] J. Jalilian-Marian, A. Kovner, A. Leonidov, and H. Weigert, Nucl. Phys. B 504, 415 (1997); Phys. Rev. D 59, 014014 (1999).

[27] E. Iancu, A. Leonidov, and L. D. McLerran, Nucl. Phys. A 692, 583 (2001); Phys. Lett. B 510, 133 (2001);

[28] F. Gelis, A. Peshier, Nucl. Phys. A 697, 879 (2002).

[29] J. Pumplin, D.R. Stump, J. Huston, H.L. Lai, P. Nadolsky, and W.K. Tung, JHEP 0207, 012 (2002).

[30] E. Iancu, K. Itakura, and S. Munier, Phys. Lett. B 590, 199 (2004).

[31] J. P. Blaizot, F. Gelis, and R. Venugopalan, Nucl. Phys. A 743, $13(2004)$

[32] E. Iancu, A. Leonidov, and L. D. McLerran, Phys. Lett. B 510 133 (2001).

[33] E. Iancu, K. Itakura, and L. McLerran, Nucl. Phys. A 724, 181 (2003).

[34] M.A. Betemps, M.B. Gay Ducati, Phys. Lett. B 636, 46 (2006).

[35] I. Arsene et al. [BRAHMS Collaboration], Phys. Rev. Lett. 93, 242303 (2004) 